\title{
Skew codes of prescribed distance or rank
}

\author{
L. Chaussade, P. Loidreau ${ }^{\dagger}$ and F. Ulmer $\stackrel{\ddagger}{\ddagger}$ \\ Final version: July 72008
}

\begin{abstract}
In this paper we generalize the notion of cyclic code and construct codes as ideals in finite quotients of non commutative polynomial rings, so called skew polynomial rings of automorphism type. We propose a method to construct block codes of prescribed rank and a method to construct block codes of prescribed distance. Since there is no unique factorization in skew polynomial rings, there are much more ideals and therefore much more codes than in the commutative case. In particular we obtain a $[40,23,10]_{4}$ code by imposing a distance and a $[42,14,21]_{8}$ code by imposing a rank, which both improve by one the minimum distance of the previously best known linear codes of equal length and dimension over those fields. There is a strong connection with linear difference operators and with linearized polynomials (or $q$-polynomials) reviewed in the first section.
\end{abstract}

\section{Galois theory of difference equations over finite fields}

Let $\left(\mathbb{F}_{q}, \theta\right)$ be a field of characteristic $p$ together with an automorphism $\theta$. A difference (or recurrence) equation over $\left(\mathbb{F}_{q}, \theta\right)$ is an equation of the form :

$$
L(y)=a_{n} \theta^{n}(y)+\ldots+a_{1} \theta(y)+a_{0} y=0 .
$$

Denote $q=p^{r}$ and $\theta(x)=x^{p^{i}}$ with $i$ in $\{0, \ldots, r-1\}$ (since the automorphism group of a field is cyclic). A finite difference field $\left(\mathbb{F}_{q^{s}}, \Theta\right)$ is a difference field extension of $\left(\mathbb{F}_{q}, \theta\right)$ if $\mathbb{F}_{q} \subseteq \mathbb{F}_{q^{s}}$ and $\Theta$ defined by $\Theta(x)=x^{p^{i}}$ is an extension of $\theta$ to an automorphism of $\mathbb{F}_{q^{s}}$. Note that even if there are several ways to extend a field automorphism, we keep the expression $\theta$ for the extension $\Theta$. A solution of the difference equation $L(y)=0$ is an element $\beta$ in a finite difference field extension of $\left(\mathbb{F}_{q}, \theta\right)$ such that $L(\beta)=0$. We call $\left(\mathbb{F}_{q}\right)^{\theta}$ the field of constants. The solution space of the difference equation $L(y)=0$ is a vector space over $\left(\mathbb{F}_{q}\right)^{\theta}$ of dimension $\leq n$. There is a difference Galois theory of difference rings

*IRMAR (UMR 6625), Université de Rennes 1, Campus de Beaulieu, F-35042 Rennes Cedex

†'IRMAR (UMR 6625) et DGA/CELAR, La Roche Marguerite, 35174 Bruz Cedex, France

${ }^{\ddagger}$ IRMAR (UMR 6625), Université de Rennes 1, Campus de Beaulieu, F-35042 Rennes Cedex 
$[3,16]$ where the existence of a difference splitting ring (of Picard-Vessiot ring) is proven under the assumption that the field of constants is algebraically closed. In our special situation of a finite coefficient field we do not want to work with an algebraically closed field of constants and we will show that if $a_{0} \neq 0$, then there always exists a finite PV-field whose dimension over the fixed field $\left(\mathbb{F}_{q}\right)^{\theta}$ is maximal. In connection with coding theory the equivalent notion of $p$-polynomial or linearized polynomial is more common $([12,15])$. In this section we recall the basic facts and connections between those notions.

To a difference equation $L(y)$ we can associate the corresponding difference operator $L=a_{n} \theta^{n}+\ldots+a_{1} \theta+a_{0}$. The set $\mathcal{L}\left(\mathbb{F}_{q}\right)$ of all difference operators over $\mathbb{F}_{q}$ can be given a ring structure $\left(\mathcal{L}\left(\mathbb{F}_{q}\right),+, \circ\right)$, where the multiplication $\circ$ is the composition of operators defined by $\left(L_{1} \circ L_{2}\right)(y)=L_{1}\left(L_{2}(y)\right)$. Starting from the finite field $\mathbb{F}_{q}$ and an automorphism $\theta$ of $\mathbb{F}_{q}$, we define a ring structure on the set

$$
\mathbb{F}_{q}[X, \theta]=\left\{a_{n} X^{n}+\ldots+a_{1} X+a_{0} \mid a_{i} \in \mathbb{F}_{q} \text { and } n \in \mathbb{N}\right\} .
$$

The addition in $\mathbb{F}_{q}[X, \theta]$ is defined to be the usual addition of polynomials and the multiplication is defined by the basic rule $X a=\theta(a) X\left(a \in \mathbb{F}_{q}\right)$ and extended to all elements of $\mathbb{F}_{q}[X, \theta]$ by associativity and distributivity (cf. $\left.[2,14]\right)$. The non commutative multiplication of skew polynomials corresponds to the composition of differential operators. The ring $\mathbb{F}_{q}[X, \theta]$ is a left and right euclidean ring whose left and right ideals are principal [14]. Left and right gcd and lcm exist in $\mathbb{F}_{q}[X, \theta]$ and can be computed using the left and right euclidean algorithm [7]. The connection between skew polynomials and difference operators is given by the map $\psi: \mathbb{F}_{q}[X, \theta] \rightarrow\left(\mathcal{L}\left(\mathbb{F}_{q}\right),+, \circ\right)$ given by

$$
\sum_{i=0}^{n} a_{i} X^{i} \mapsto \sum_{i=0}^{n} a_{i} \theta^{i}
$$

which is a morphism of rings. In the following we will sometime associate to a difference operator $L=\sum_{i=0}^{n} a_{i} \theta^{i}$ of the difference equation $L(y)=\sum_{i=0}^{n} a_{i} \theta^{i}(y)$ the skew polynomial $f_{L}=\sum_{i=0}^{n} a_{i} X^{i} \in \mathbb{F}_{q}[X, \theta]$.

Suppose that $q=q_{0}^{t}$ and consider $\theta \in \operatorname{Aut}\left(\mathbb{F}_{q}\right)$ given by $a \mapsto a^{q_{0}}$. The fixed field $\left(\mathbb{F}_{q}\right)^{\theta}$ of $\theta$ is $\mathbb{F}_{q_{0}}$. It is classical (Section 5 of [12] or " $p$-polynomials " in [15]) to associate to $L(y)$ the affine linearized polynomial:

$$
\ell(Y)=a_{n} Y^{\left(q_{0}\right)^{n}}+\ldots+a_{1} Y^{q_{0}}+a_{0} Y \in \mathbb{F}_{q}[Y] .
$$

This amounts to express the action of the automorphism, and therefore there is a bijective correspondance between solutions of $L(y)=0$ in a finite difference field extension and distinct roots of the classical commutative polynomial $\ell(Y)=0$.

Definition 1 We call multiplicity of a solution, $\beta$, of $L(y)=0$ the order of $\beta$ as a root of the associated linearized polynomial $\ell(Y)$.

Example 1 In the example $L(y)=\theta^{n}(y), 0$ is a solution of multiplicity $\left(q_{0}\right)^{n}$. 
The following is a reformulation of [12], Theorem 3.50:

Lemma 1 Let $\theta$ be an automorphism of $\mathbb{F}_{q}$ defined by $a \mapsto a^{q_{0}}$, denote $\left(\mathbb{F}_{q}\right)^{\theta}=\mathbb{F}_{q_{0}}$ the fixed field of $\theta$ and $L(y)=\sum_{i=0}^{n} a_{i} \theta^{i}(y)$ with $a_{i} \in \mathbb{F}_{q}$. Each solution of $L(y)=0$ has multiplicity $\left(q_{0}\right)^{k}$ where $k=\min \left\{i, a_{i} \neq 0\right\}$.

Proof. The first part is proven in [12], Theorem 3.50 and only the claim on the multiplicity has to be verified. First we note that the derivative of $\ell(Y)$ is $\ell^{\prime}(Y)=a_{0}$, so that if $a_{0} \neq 0$ then all the solutions of $\ell(Y)=0$ have multiplicity 1 .

Suppose that $q=q_{0}^{t}$ and that $a_{0}=a_{1}=\ldots=a_{k-1}=0$ and $a_{k} \neq 0$, then

$$
\ell(Y)=\sum_{i=k}^{n} a_{i} Y^{q_{0}^{i}}=\sum_{i=k}^{n} a_{i}^{q_{0}^{t k}} Y^{q_{0}^{i}} .
$$

The last equality is true because $a_{i}$ belongs to $\mathbb{F}_{q_{0}^{t}}=\mathbb{F}_{q}$. We get

$$
\ell(Y)=\sum_{i=k}^{n} a_{i}^{q_{0}^{t k}} Y^{q_{0}^{i}}=\left[\sum_{i=k}^{n} a_{i}^{q_{0}^{(t-1) k}} Y^{q_{0}^{i-k}}\right]_{0}^{q_{0}^{k}}
$$

By hypothesis the polynomial in brackets has his derivate $a_{k}^{q_{0}^{(r-1) k}} \neq 0$ and thus all its roots have order 1 . Therefore all the solutions of $\ell(Y)$ have multiplicity $q_{0}^{k}$.

The following theorem shows a link between the dimension of the vector space of solutions and the coefficients of $L$.

Theorem 1 (cf. [15], Theorem 5) Let $\theta$ be an automorphism of $\mathbb{F}_{q}$ defined by $a \mapsto a^{q_{0}}$, denote $\left(\mathbb{F}_{q}\right)^{\theta}=\mathbb{F}_{q_{0}}$ the fixed field of $\theta$ and $L(y)=\sum_{i=0}^{n} a_{i} \theta^{i}(y)$ with $a_{i} \in \mathbb{F}_{q}$. There exists a finite field $\mathbb{F}_{q^{s}}$ which contains all the roots of $\ell_{L}(Y)=0$ and the $\left(\mathbb{F}_{q}\right)^{\theta}$-subspace of $\mathbb{F}_{q^{s}}$ spanned by those roots is of dimension $n-\min \left\{i, a_{i} \neq 0\right\}$. In particular if $a_{0} \neq 0$ then the smallest field $\mathbb{F}_{q^{s}}$ is a difference splitting field (or Picard-Vessiot field) of $L(y)=0$.

Proof. We compute the dimension of the vector space of solutions in a finite field extension by counting the solutions of the associated linearized polynomial $\ell(Y)$. Since $\ell(Y)$ is a polynomial of degree $q_{0}^{n}$, it has $q_{0}^{n}$ roots counted with multiplicity in a decomposition field. If $\min \left\{a_{i}, a_{i} \neq 0\right\}=k$ then the proof of lemma shows that $L(y)$ has $q_{0}^{n}$ solutions of multiplicity $q_{0}^{k}$, it follows that the dimension of the vector space spaned by the solutions over $\mathbb{F}_{q_{0}}$ has dimension $n-k$.

Example 2 Let $\theta(x)=x^{q_{0}}$, then

1. The solution space of $L(y)=\theta^{n}(y)-y$ is $\mathbb{F}_{\left(q_{0}\right)^{n}}$. It is a vector space of dimension $n$ over $\mathbb{F}_{q_{0}}\left(\right.$ here $\left.a_{0}=-1 \neq 0\right)$.

2. The solution space of $L(y)=\theta^{n}(y)$ is $\{0\}$. It is a vector space of dimension 0 $\left(a_{0}=\ldots a_{n-1}=0\right.$ so $\left.\min \left\{a_{i}, a_{i} \neq 0\right\}=n\right)$.

3. The solution space of $L(y)=\theta^{n}(y)-\theta^{n-1}(y)$ is $\mathbb{F}_{q_{0}}$. To see this, note that if $\alpha$ is a solution, then $\theta(\alpha)-\alpha=0$ and therefore $\alpha \in \mathbb{F}_{q_{0}}$. In this case the multiplicity of each solution is $\left(q_{0}\right)^{n-1}$. 


\section{Coding with skew polynomials}

If $m$ is the order of $\theta$, then the polynomial $f \in \mathbb{F}_{q}[X, \theta]$ generates a two-sided ideal in $\mathbb{F}_{q}[X, \theta]$ if and only if $f$ is of the form $X^{e} \cdot \rho$, where $\rho \in\left(\mathbb{F}_{q}\right)^{\theta}\left[X^{m}\right]$ is a central element of $\mathbb{F}_{q}[X, \theta]$ (cf.[1], Theorem 3.2.16). In particular a left or right ideal in $\mathbb{F}_{q}[X, \theta]$ generated by a central element is a two sided ideal. If $I$ is a two sided ideal in $\mathbb{F}_{q}[X, \theta]$ then $I$ is generated by a polynomial $f$ of some degree $n$ with the above property and, by the correspondance of ideals, the left ideals in $\mathbb{F}_{q}[X, \theta] /(f)$ are principal ideals, each generated by a right divisor $g$ of $f$. To the element $a(X)=a_{n-1} X^{n-1}+\ldots+a_{1} X+a_{0}$ in $\mathbb{F}_{q}[X, \theta] /(f)$ we associate the word $a=\left(a_{0}, a_{1}, \ldots, a_{n-1}\right) \in \mathbb{F}_{q}^{n}$. The elements $a(X)$ of $\mathbb{F}_{q}[X, \theta] /(f)$ that belong to a left ideal in $\mathbb{F}_{q}[X, \theta] /(f)$ generated by a right divisor $g$ of $f$ form a linear subspace of $\left(\mathbb{F}_{q}\right)^{n}$, which is a code of length $n$ and of dimension $k=n-\operatorname{deg}(g)$. More precisely :

Definition 2 (cf. [6]) Let $f \in \mathbb{F}_{q}[X, \theta]$ be of degree $n$. If $I=(f)$ is a two sided ideal of $\mathbb{F}_{q}[X, \theta]$, then a $\theta$-code $\mathcal{C}$ consists of codewords $a=\left(a_{0}, a_{1}, \ldots, a_{n-1}\right)$ that are coefficient tuples of elements $a(X)=a_{n-1} X^{n-1}+\ldots+a_{1} X+a_{0}$ of a left ideal of $\mathbb{F}_{q}[X, \theta] / I$. In this case the elements $a(X)$ are left multiples of a right divisor $g$ of $f$. We will focus on two special cases :

1. If $f$ generates a two-sided ideal in $\mathbb{F}_{q}[X, \theta]$, then we call the code corresponding to the left ideal $(g) /(f)$ a $\theta$-code.

2. If $m=|\langle\theta\rangle|$ divides $n$ and $f=X^{n}-1$, then we call the $\theta$-code corresponding to the left ideal $(g) /\left(X^{n}-1\right)$ a $\theta$-cyclic code.

Note that codewords of skew codes can be identified with the multiples of the generating skew polynomial $g \in \mathbb{F}_{q}[X, \theta]$, but not with the solutions of the corresponding difference equation. If $g=g_{r} X^{r}+\ldots+g_{1} X+g_{0}$ divides a polynomial $f \in \mathbb{F}_{q}[X, \theta]$ of degree $n$ such that $(f)$ is a two-sided ideal, then the generator matrix of the $\theta$-code generated by $g$ is given by

$$
G=\left(\begin{array}{ccccccc}
g_{0} & \ldots & g_{r-1} & g_{r} & 0 & \ldots & 0 \\
0 & \theta\left(g_{0}\right) & \ldots & \theta\left(g_{r-1}\right) & \theta\left(g_{r}\right) & \ldots & 0 \\
0 & \ddots & \ddots & \ddots & \ddots & \ddots & \vdots \\
0 & & & & & & \\
0 & \ldots & 0 & \theta^{n-r-1}\left(g_{0}\right) & \ldots & \theta^{n-r-1}\left(g_{r-1}\right) & \theta^{n-r-1}\left(g_{r}\right)
\end{array}\right)
$$

The length of the code is the degree $n$ of $f$ and its dimension is $k=n-r$. If the Hamming distance of the code is $d$, then the code is said to be an $[n, k, d]$-code. The smallest length of a code that a skew polynomial $g \in \mathbb{F}_{q}[X, \theta]$ does generate is the degree $n$ of the generator $g^{*}$ of the largest two-sided ideal $\left(g^{*}\right) \subset \mathbb{F}_{q}[X, \theta]$ (i.e. such that $g^{*}$ is of smallest degree) contained in the left ideal $(g) \subset \mathbb{F}_{q}[X, \theta]$. If $m$ is the order of $\theta$, then the polynomial $g^{*}$ is called the bound of $g$ and must be of the form $X^{e} \cdot \rho$, where $\rho \in\left(\mathbb{F}_{q}\right)^{\theta}\left[X^{m}\right]$ is a central element of $\mathbb{F}_{q}[X, \theta]$ (cf.[1], Theorem 3.2.16). In order to compute the bound $g^{*}$, we can use the algorithm described in the proof of ([6], Lemma 10). 


\section{Skew codes with constructed rank}

If $f \in \mathbb{F}_{q}[X, \theta]$ generates a two sided ideal and $f=h \cdot g$, then any codeword $c$ of the $\theta$-code $(g) /(f) \subset \mathbb{F}_{q}[X, \theta] /(f)$ can be uniquely represented by a skew-polynomial of $\mathbb{F}_{q}[X, \theta]$ of degree $n-1$. Therefore it can be represented as a $n$-dimensional vector with components in $\mathbb{F}_{q}$. Since $\left[\mathbb{F}_{q}:\left(\mathbb{F}_{q}\right)^{\theta}\right]=m$ (where $m$ is the order of $\theta$ ), it can also be represented as a $m \times n$ matrix with coefficients over the field of constants. The rank of this matrix is called the rank of codeword $c$ and is denoted $\mathrm{Rk}(c)$. It is clearly independent of the chosen basis of $\mathbb{F}_{q} /\left(\mathbb{F}_{q}\right)^{\theta}$.

Where the Hamming metric is the suitable metric for correcting errors occuring in the Binary Symmetric Channel, rank metric is suitable for correcting criss-cross errors, or errors occuring along vector spaces.

For example let $c=(0,0, \gamma, 0, \beta)$, where $\gamma \in \mathbb{F}_{q}^{*}$ and $\beta \in \mathbb{F}_{q}^{*}$. The Hamming weight of $\mathbf{c}$ is equal to 2. The rank of $\mathbf{c}$ is equal to 1 or 2 . It has rank 1 if and only if there exists $\lambda \in\left(\mathbb{F}_{q}\right)^{\theta} \backslash\{0\}$ such that $\gamma=\lambda \beta$. We can thus define the minimum rank distance of a linear code by

Definition 3 The minimum rank distance of a code $\mathcal{C}$ of length $n$ over $\left(\mathbb{F}_{q}\right)^{\theta}$ is the integer d such that

$$
d=\min _{c \in \mathcal{C} \backslash\{0\}}(R k(c))
$$

For a more precise description of rank metric in terms of coding theory, see [9].

Proposition 1 Let $m$ be the order of $\theta \in A u t\left(\mathbb{F}_{q}\right), g \in \mathbb{F}_{q}[X, \theta]$ and $L_{g}(y)=0$ be the associated differential equation. Suppose that there exist integers $n$ and $\delta$ with $\tau \geq \delta \geq 1$ and an element $\beta$ in the splitting field $\mathbb{F}_{q^{s}}$ of $L_{g}(y)$ such that

- $\beta, \theta(\beta), \ldots, \theta^{\tau-1}(\beta)$ are linearly independent over $\left(\mathbb{F}_{q}\right)^{\theta}$.

- for $i \in\{0, \ldots, \delta-1\}$, the element $\theta^{i}(\beta)$ is a solution of $L_{g}(y)=0$, i.e. $L_{g}\left(\theta^{i}(\beta)\right)=0$.

Then, for all skew polynomials $\in\left(\mathbb{F}_{q}\right)^{\theta}\left[X^{m}\right]$ of degree $\tau$ in the center of $\mathbb{F}_{q}[X, \theta]$ which are right divisible by $g$, the code $(g) /(f)$ has a minimum rank distance $\geq \delta+1$. Therefore its minimum distance is at least $\delta+1$.

Proof. Let $c \in(g) /(f)$ be a non-zero codeword of rank $\tau$ over $\left(\mathbb{F}_{q}\right)^{\theta}$. The coefficients of $c$ form a $n$-dimensional vector $\left(c_{0}, \ldots, c_{n-1}\right) \in\left(\mathbb{F}_{q}\right)^{n}$ of rank $\tau$ over $\left(\mathbb{F}_{q}\right)^{\theta}$. Hence, there exists $U \in M_{\tau \times n}\left(\left(\mathbb{F}_{q}\right)^{\theta}\right)$ of rank $\tau$ and $C_{1}, \ldots, C_{\tau} \in\left(\mathbb{F}_{q}\right)^{\tau}$ linearly independent over $\left(\mathbb{F}_{q}\right)^{\theta}$ such that

$$
\left(c_{0}, \ldots, c_{n-1}\right)=\left(C_{1}, \ldots, C_{\tau}\right) U
$$

Since a codeword $c$ is a left multiple $h \cdot g$ of $g$ and since multiplication in $\mathbb{F}_{q}[X, \theta]$ corresponds to the composition of the differential operators, we have $L_{c}(y)=L_{h}\left(L_{g}(y)\right)$. Therefore any solution $\gamma$ of $L_{g}(y)=0$ is also a solution of $L_{c}(y)=0$ and there exists a basis 
of the space of solutions of the associated difference equation $L_{g}(y)=0$ of the form $\left(\beta, \ldots, \theta^{\delta-1}(\beta), \gamma_{\delta}, \ldots, \gamma_{k-1}\right)$. This shows that

$$
\left(c_{0}, \ldots, c_{n-1}\right)\left(\begin{array}{cccccc}
\beta & \ldots & \theta^{\delta-1}(\beta) & \gamma_{\delta} & \ldots & \gamma_{k-1} \\
\theta(\beta) & \ldots & \theta^{\delta}(\beta) & \theta\left(\gamma_{\delta}\right) & \ldots & \theta\left(\gamma_{k-1}\right) \\
\vdots & \ddots & \vdots & \vdots & \ddots & \vdots \\
\theta^{n-1}(\beta) & \cdots & \theta^{n+\delta-2}(\beta) & \theta^{n-1}\left(\gamma_{\delta}\right) & \ldots & \theta^{n-1}\left(\gamma_{k-1}\right)
\end{array}\right)=0
$$

If we define

$$
\forall i=1, \ldots, \tau, \quad u_{i}(\beta) \stackrel{\text { def }}{=} \sum_{j=0}^{n} U_{i j} \theta^{i}(\beta),
$$

and we replace $\left(c_{0}, \ldots, c_{n-1}\right)$ by the expression $(2)$, using the fact that the coefficients of $U$ lie in the field of constants, we obtain :

$$
\left(C_{1}, \ldots, C_{\tau}\right)\left(\begin{array}{cccccc}
u_{1}(\beta) & \ldots & \theta^{\delta-1}\left(u_{1}(\beta)\right) & u_{1}\left(\gamma_{\delta}\right) & \ldots & u_{1}\left(\gamma_{k-1}\right) \\
u_{2}(\beta) & \cdots & \theta^{\delta-1}\left(u_{2}(\beta)\right) & u_{2}\left(\gamma_{\delta}\right) & \ldots & u_{2}\left(\gamma_{k-1}\right) \\
\vdots & \ddots & \vdots & \vdots & \ddots & \vdots \\
u_{\tau}(\beta) & \cdots & \theta^{\delta-1}\left(u_{\tau}(\beta)\right) & u_{\tau}\left(\gamma_{\delta}\right) & \ldots & u_{\tau}\left(\gamma_{k-1}\right)
\end{array}\right)=0
$$

Since $U$ has rank $\tau$, and since $\beta, \ldots, \theta^{N-1}(\beta)$ are linearly independent over the field of constants, this implies that $u_{1}(\beta), \ldots, u_{\tau}(\beta)$ are linearly independent over the field of constants and therefore the first $\delta$ columns of the matrix of the system are linearly independent. Hence if $\tau \leq \delta$, the previous equation has no non-zero solution. Therefore, the minimum rank distance of $(g) /(f)$ is at least $\delta+1$.

The above skew code of prescribed rank $\delta$ in the previous proposition will be denoted $\mathcal{C}_{\beta, \ldots, \theta^{\delta-1}(\beta)}$. In order to construct all codes $\mathcal{C}_{\beta, \ldots, \theta^{\delta-1}(\beta)}$ of length $n$, smaller than a given bound $N$, which are of prescribed rank $\delta$ defined over $\mathbb{F}_{q}$, we proceed as follows:

1. Consider in turn all non trivial automorphisms $\theta \in \operatorname{Aut}\left(\mathbb{F}_{q}\right)$. Through this choice, the ring $\mathbb{F}_{q}[X, \theta]$ and therefore the constant field $\left(\mathbb{F}_{q}\right)^{\theta}$ of order $q_{0}$ are determined.

2. Consider in turn any $\beta$ in a field extension $\mathbb{F}_{q^{s}}$ of $\mathbb{F}_{q}$ with $s \leq q^{N}$ (the degree of a linearized polynomial over $\mathbb{F}_{q}$ of some $f \in\left(\mathbb{F}_{q}\right)^{\theta}\left[X^{|\theta|}\right]$ of degree $n \leq N$ where $|\theta|$ denotes the order of the automorphism $\theta$. Compute the longest sequence $\beta, \theta(\beta), \ldots, \theta^{\tau-1}(\beta)$ in $\mathbb{F}_{q^{s}}$ that is linearly independent over $\left(\mathbb{F}_{q}\right)^{\theta}$. Any subsequence $\beta, \ldots, \theta^{j-1}(\beta)$ could correspond to a $\mathcal{C}_{\beta, \ldots, \theta^{\delta-1}(\beta)}$ code, but we need to construct the code (i.e. find the generating polynomial $g$ with the property that the associated difference equation $L_{g}(y)=0$ has $\beta, \ldots, \theta^{\delta-1}(\beta)$ among its solutions) in order to find $\delta$ and we need to verify that its length $n$ (the degree of the bound of $g$ ) is less than $\tau$ (we will see that the length $n$ must in fact be $\tau$ )

(a) Denote $\sigma$ a generator of $\operatorname{Aut}\left(\mathbb{F}_{q^{s}} / \mathbb{F}_{q}\right)$. If $\sigma$ is not a power of the extension of $\theta$ to $\Theta \in \operatorname{Aut}\left(\mathbb{F}_{q^{s}}\right)$, then the fixed field of $\Theta$ is no longer contained in $\mathbb{F}_{q}$ and we stop the computation for this $\theta$. Otherwise construct the smallest $\sigma$-invariant $\left(\mathbb{F}_{q}\right)^{\theta}$-subspace $V_{g}$ of $\mathbb{F}_{q^{s}}$ containing $\beta, \ldots, \theta^{j-1}(\beta)$. 
(b) Construct the skew polynomial $g \in \mathbb{F}_{q}[X, \theta]$ so that the corresponding difference operator $L_{g}(y)=0$ has $V_{g}$ as solution space. We will show that $g$ is defined over $\mathbb{F}_{q}$ if and only if $V_{g}$ is $\sigma$-invariant.

(c) Using the algorithm presented in ([6], Lemma 10), compute the bound $f$ of $g$. Verify that its degree $n$ of $f$ is less than $\tau$.

In practice, the computation of the bound is not necessary, since we will show that $g$ does generate a $\mathcal{C}_{\beta, \ldots, \theta^{\delta-1}(\beta)}$ code if and only if the $\left(\mathbb{F}_{q}\right)^{\theta}$-subspace spanned by $\beta, \theta(\beta), \ldots, \theta^{\tau-1}(\beta)$ is the solution space of the difference operator corresponding to a central polynomial $f \in$ $\left(\mathbb{F}_{q}\right)^{\theta}\left[X^{|\theta|}\right]$. Therefore we must have $n=\tau$.

Note that Gabidulin codes correspond to the case where the length $n$ is maximal, i.e. $n=\tau=\left[\mathbb{F}_{q^{s}}:\left(\mathbb{F}_{q}\right)^{\theta}\right]$. In this case $\tau$ is the order of $\operatorname{Aut}\left(\left(\mathbb{F}_{q^{s}} /\left(\mathbb{F}_{q}\right)^{\theta}\right)\right.$ and the bound $f$ must be $X^{\tau}-1$. We will be particularly interested in codes that are not of this type.

In the following we review the classical result showing how to reconstruct a difference equation from a given solution space or a fundamental set of solutions $\left\{y_{1}, \ldots, y_{n}\right\}$ (see [15], Theorem 7). This is always possible if if the constant term $a_{0}$ is non zero. The case $a_{0}=0$ is less interesting since such a code is obtained from a code with $a_{0} \neq 0$ by adding columns of zeros to the generator matrix ([6], Proposition 1). The solutions $y$ of such a difference equation are precisely the $y$ that are linearly dependent with $\left\{y_{1}, \ldots, y_{n}\right\}$, i.e. such that the Casoratian

$$
\operatorname{Cas}\left(y_{1}, \ldots, y_{n}, y\right)=\left|\begin{array}{ccccc}
y_{1} & y_{2} & \ldots & y_{n} & y \\
\theta\left(y_{1}\right) & \theta\left(y_{2}\right) & \ldots & \theta\left(y_{n}\right) & \theta(y) \\
\theta^{2}\left(y_{1}\right) & \theta^{2}\left(y_{2}\right) & \ldots & \theta^{2}\left(y_{n}\right) & \theta^{2}(y) \\
\ldots & \ldots & \ldots & \ldots & \ldots \\
\theta^{n}\left(y_{1}\right) & \theta^{n}\left(y_{2}\right) & \ldots & \theta^{n}\left(y_{n}\right) & \theta^{n}(y)
\end{array}\right|=0 .
$$

We denote by $\mathrm{Cas}_{i}$ the above determinant where the last column and the i-th row have been deleted. Expanding the determinant along the last column gives :

$$
L_{y_{1}, \ldots, y_{n}}(y)=\theta^{n}(y)+\sum_{i=0}^{n-1} \frac{\operatorname{Cas}_{i+1}\left(y_{1}, \ldots, y_{n}\right)}{\operatorname{Cas}\left(y_{1}, \ldots, y_{n}\right)} \theta^{i}(y)
$$

This allows to express the coefficients of a difference equation from a fundamental set of solutions and is therefore analogous to the result about symmetric functions. Next we want to insure that the code we are constructing is defined over $\mathbb{F}_{q}$.

Lemma 2 Be $\theta \in \operatorname{Aut}\left(\mathbb{F}_{q}\right)$, $\sigma$ a generator of the Galois group of $\mathbb{F}_{q^{s}} / \mathbb{F}_{q}$ and $y_{i} \in \mathbb{F}_{q^{s}}$ such that $y_{1}, \cdots, y_{n}$ are linearly independent over $\left(\mathbb{F}_{q}\right)^{\theta}$. The difference equation $L_{y_{1}, \ldots, y_{n}}(y)$ is defined over $\mathbb{F}_{q}$ if and only if $y_{1}, \cdots, y_{n}$ span a $\left(\mathbb{F}_{q}\right)^{\theta}$-vector space that is invariant under $\sigma$.

PROOF. In one direction we have to show that the coefficients of $L_{y_{1}, \ldots, y_{n}}(y)$ belong to $\mathbb{F}_{q}:$

$$
\sigma\left(\frac{\operatorname{Cas}_{i+1}\left(y_{1}, \ldots, y_{n}\right)}{\operatorname{Cas}\left(y_{1}, \ldots, y_{n}\right)}\right)=\frac{\operatorname{det}(\sigma) \cdot \operatorname{Cas}_{i+1}\left(y_{1}, \ldots, y_{n}\right)}{\operatorname{det}(\sigma) \cdot \operatorname{Cas}\left(y_{1}, \ldots, y_{n}\right)}=\frac{\operatorname{Cas}_{i+1}\left(y_{1}, \ldots, y_{n}\right)}{\operatorname{Cas}\left(y_{1}, \ldots, y_{n}\right)}
$$


and therefore the coefficients of $L_{y_{1}, \ldots, y_{n}}(y)$ belong to $\mathbb{F}_{q}$, the fixed field of $\sigma$. The proof of the above is similar to the differential case using the fact that $\sigma$ and (the extension of) $\theta$ commute (cf. [17], p27, Exercice 4c).

For the converse, suppose that $L_{y_{1}, \ldots, y_{n}}(y)=\sum_{i=0}^{n} a_{i} \theta^{i}(y)$ with $a_{i} \in \mathbb{F}_{q}$. Since $\sigma$ and (the extension to $\mathbb{F}_{q^{s}}$ of ) $\theta$ commute, we get that :

$$
0=\sigma\left(\sum_{i=0}^{n} a_{i} \theta^{i}\left(y_{j}\right)\right)=\sum_{i=0}^{n} a_{i} \theta^{i}\left(\sigma\left(y_{j}\right)\right),
$$

which shows that the space spanned by $y_{1}, \cdots, y_{n}$ is invariant under $\sigma$.

We are now in a position to compute the smallest generating polynomial $g$ having the roots $\beta, \theta(\beta), \ldots, \theta^{\delta-1}(\beta)$ and to guarantee that it is defined over $\mathbb{F}_{q}$. For that we consider a basis $y_{1}, \ldots, y_{\ell}$ of the space spanned by $\beta, \theta(\beta), \ldots, \theta^{\delta-1}(\beta)$. We add to this set of linearly independent vectors any image under the generator $\sigma$ of $\operatorname{Aut}\left(\mathbb{F}_{q^{s}} / \mathbb{F}_{q}\right)$ of the basis vectors, until we obtain a basis $y_{1}, \ldots, y_{r}$ that is stable under $\sigma$. According to the above Lemma, the corresponding difference equation $L_{y_{1}, \ldots, y_{r}}(y)$ is defined over $\mathbb{F}_{q}$ and has the solutions $\beta, \theta(\beta), \ldots, \theta^{\delta-1}(\beta)$.

We now focus on the length of the code generated by the skew polynomial $g$ corresponding to the difference operator $L_{y_{1}, \ldots, y_{r}}(y)$. We need to find a bound $f$ of $g$.

Lemma 3 Let $\beta \in \mathbb{F}_{q^{s}}$ and $t \in \mathbb{N}$ such that $\beta, \ldots, \theta^{\tau-1}(\beta)$ is the longest sequence that is linearly independent over $\left(\mathbb{F}_{q}\right)^{\theta}$. Denote $g$ the generator of the code $\mathcal{C}_{\beta, \ldots, \theta^{\delta-1}(\beta)}$. Then the bound $g^{*}$ of $g$ must be a central polynomial $\rho \in\left(\mathbb{F}_{q}\right)^{\theta}\left[X^{m}\right]$ of $\mathbb{F}_{q}[X, \theta]$ of degree $n=\tau$ with the property that the solution space of the associated difference operator $L_{\rho}(y)$ is spanned by $\beta, \theta(\beta), \ldots, \theta^{n-1}(\beta)$.

Proof. Since $g^{*}$ is a bound of $g$ we have $g^{*}=h \cdot g$ in $\mathbb{F}_{q}[X, \theta]$. Using difference operators we have $L_{g^{*}}=L_{h} \circ L_{g}$. This means that $\beta$ is a solution of $L_{g^{*}}$. Since $g^{*}$ generates a two-sided ideal, it must be of the form $X^{e} \cdot \rho$, where $\rho \in\left(\mathbb{F}_{q}\right)^{\theta}\left[X^{m}\right]$ is a central element of $\mathbb{F}_{q}[X, \theta]$ (cf.[1], Theorem 3.2.16). In particular the coefficients of $L_{g^{*}}$ are in $\left(\mathbb{F}_{q}\right)^{\theta}$, showing that $L_{g^{*}}\left(\theta^{j}(\beta)\right)=\theta^{j}\left(L_{g^{*}}(\beta)\right)=0$ for $j$ in $\{0, \ldots, \tau-1\}$. Therefore $L_{g^{*}}$ has at least $\tau$ linearly independent solutions and it follows that the degree of $g^{*}$ is at least $\tau$. Since the degree of $g^{*}$ is at most $\tau$ by definition of $\mathcal{C}_{\beta, \ldots, \theta^{\delta-1}(\beta)}$, the degree of $g^{*}$ must be $n=\tau$ and the solution space of $g^{*}$ is spanned by $\beta, \theta(\beta), \ldots, \theta^{\tau-1}(\beta)$.

Since $L_{g^{*}}(y)$ is a difference operator of order $n=\tau$ and has a solution space of dimension $\tau$ in a finite field extension of $\mathbb{F}_{q}$, Lemma 1 shows that the constant term of $g^{*}$ must be non zero. Therefore $e=0$ and that $g^{*}=\rho$ is a central element belonging to $\left(\mathbb{F}_{q}\right)^{\theta}\left[X^{m}\right]$.

The previous discussion leads to the following algorithm to construct all codes $\mathcal{C}_{\beta, \ldots, \theta^{\delta-1}(\beta)}$ of length $n$ smaller than a given bound $N$ which are of prescribed rank $\delta \geq \Delta$ defined over $\mathbb{F}_{q}$. We proceed as follows :

1. Consider in turn all non trivial automorphisms $\theta \in \operatorname{Aut}\left(\mathbb{F}_{q}\right)$. At this point we fixed the ring $\mathbb{F}_{q}[X, \theta]$ and therefore the constant field $\left(\mathbb{F}_{q}\right)^{\theta}$ of order $q_{0}$. 
2. Consider in turn any $\beta$ in a field extension $\mathbb{F}_{q^{s}}$ of $\mathbb{F}_{q}$ with $s \leq q^{n}$ with the property that a generator $\sigma$ of $\operatorname{Aut}\left(\mathbb{F}_{q^{s}} / \mathbb{F}_{q}\right)$ is a power of the extension of $\theta$ to $\Theta \in \operatorname{Aut}\left(\mathbb{F}_{q^{s}}\right)$.

3. For each $\beta$ compute the longest sequence $\beta, \theta(\beta), \ldots, \theta^{n-1}(\beta)$ in $\mathbb{F}_{q^{s}}$ that is linearly independent over $\left(\mathbb{F}_{q}\right)^{\theta}$ and check that the skew polynomial $f$ corresponding to $L_{\beta, \theta(\beta), \ldots, \theta^{n-1}(\beta)}(y)$, which by construction belongs to $f \in\left(\mathbb{F}_{q}\right)^{\theta}[X, \theta]$, is central, i.e. belongs to $\left(\mathbb{F}_{q}\right)^{\theta}\left[X^{|\theta|}\right]$.

4. If $f \in\left(\mathbb{F}_{q}\right)^{\theta}\left[X^{|\theta|}\right]$, then for any subsequence $\beta, \ldots, \theta^{j-1}(\beta)$, construct the smallest $\sigma$-invariant $\left(\mathbb{F}_{q}\right)^{\theta}$-subspace $V_{j}$ of $\mathbb{F}_{q^{s}}$ containing $\beta, \ldots, \theta^{j-1}(\beta)$, where $\sigma$ is a generator of $\operatorname{Aut}\left(\mathbb{F}_{q^{s}} / \mathbb{F}_{q}\right)$. In order to construct a basis of $V_{j}$, it is sufficient to to start with an $\left(\mathbb{F}_{q}\right)^{\theta}$-basis of the space spanned by $\beta, \ldots, \theta^{j-1}(\beta)$ and to iteratively add those images under $\sigma$, that are not linearly dependent with the space constructed so far, until the resulting space is $\sigma$-invariant.

5. The resulting skew polynomial $g \in \mathbb{F}_{q}[X, \theta]$ will be a skew code of prescribed rank $\delta$, where $\delta$ is the largest integer such that $\left\{\beta, \ldots, \theta^{\delta-1}(\beta)\right\} \subset V_{j}$.

We recall that in coding theory a $[n, k, d]$-code is a linear code of length $n$, dimension $k$ and minimum distance $d$.

Example 3 Consider $\mathbb{F}_{q}=\mathbb{F}_{4}, \theta(x)=x^{2}$ and $s=6$. This means that we will use elements $\beta \in \mathbb{F}_{4^{6}}$ in order to construct codes over $\mathbb{F}_{4}$. We denote by $\alpha$ the generator of $\mathbb{F}_{4^{6}}$ and $w$ the generator of $\mathbb{F}_{4}$ given by MAGMA.

1. Consider $\beta=\alpha^{3688}$. The longest sequence which is linearly independent over $\mathbb{F}_{2}=$ $\left(\mathbb{F}_{4}\right)^{\theta}$ is $\beta, \theta(\beta), \ldots, \theta^{7}(\beta)$. Therefore, if we obtain a skew code of constructed rank, this code must be of length $n=8$. Using the Casoratian determinant we compute $L_{\beta}(y)=\left(\theta^{8}+\theta^{6}+\theta^{2}+1\right)(y)$. Since the associated operator $f_{L}=X^{8}+X^{6}+$ $X^{2}+1 \in \mathbb{F}_{2}[X, \theta]$ is a central polynomial (i.e. $f \in \mathbb{F}_{2}\left[X^{2}\right]$ ), we will be able to construct a skew code over $\mathbb{F}_{4}$ of constructed rank using this $\beta \in \mathbb{F}_{4^{6}}$. We start with $j=1$, and compute the smallest $\mathbb{F}_{2}$-space $V_{1}$ containing $\{\beta\}$ which is stable under the generator $\sigma: x \mapsto x^{4}$ of $A u t\left(\mathbb{F}_{4^{6}} / \mathbb{F}_{4}\right)$. A basis of $V_{1}$ is $\left\{\beta, \theta^{2}(\beta), \theta^{4}(\beta), \theta^{6}(\beta)\right\}$ (note that $\left.\sigma=\theta^{2}\right)$. Using the Casarotian determinant, we compute the skew polynomial $g=X^{4}+\alpha^{2730} X^{3}+X^{2}+X+1=X^{4}+w X^{3}+X^{2}+X+1$ associated with the corresponding difference operator having the solution space $V_{1}$. Since the length of the skew code is 4 , the generator matrix of the code is:

$$
\left(\begin{array}{cccccccc}
1 & 1 & 1 & w^{2} & 1 & 0 & 0 & 0 \\
0 & 1 & 1 & 1 & w & 1 & 0 & 0 \\
0 & 0 & 1 & 1 & 1 & w^{2} & 1 & 0 \\
0 & 0 & 0 & 1 & 1 & 1 & w & 1
\end{array}\right)
$$

We obtain a $[8,4,4]$ skew code over $\mathbb{F}_{4}$ of prescribed rank 2 . This code maps to a $[16,8,4]$ code over $\mathbb{F}_{2}$. 
2. Consider $\beta=\alpha^{1444}$. The longest sequence which is linearly independent over $\mathbb{F}_{2}=$ $\left(\mathbb{F}_{4}\right)^{\theta}$ is $\beta, \theta(\beta), \ldots, \theta^{11}(\beta)$. This means that $\beta$ generates a normal basis and that the bound must be $f=X^{12}-1$ (which is always a bound in this case, because the order of $\theta \in \operatorname{Aut}\left(\mathbb{F}_{4^{6}} / \mathbb{F}_{2}\right)$ is 12$)$. The resulting code will be a Gabidulin code. A basis of the smallest $\mathbb{F}_{2}$-space $V_{1}$ containing $\{\beta\}$, which is stable under the generator $\sigma: x \mapsto x^{4}$ of $A u t\left(\mathbb{F}_{4^{6}} / \mathbb{F}_{4}\right)$, is $\left\{\beta, \theta^{2}(\beta), \theta^{4}(\beta), \theta^{6}(\beta), \theta^{8}(\beta), \theta^{10}(\beta)\right\}$. Using the Casarotian determinant, we compute the skew polynomial

$$
\begin{aligned}
g & =X^{6}+\alpha^{1365} X^{5}+\alpha^{1365} X^{4}+\alpha^{1365} X^{3}+\alpha^{2730} X^{2}+\alpha^{1365} X+1 \\
& =X^{6}+w^{2} X^{5}+w^{2} X^{4}+w^{2} X^{3}+w X^{2}+w^{2} X+1 .
\end{aligned}
$$

Since the length of the skew code is 12 , the generator matrix of the code is :

$$
\left(\begin{array}{cccccccccccc}
1 & w^{2} & w & w^{2} & w^{2} & w^{2} & 1 & 0 & 0 & 0 & 0 & 0 \\
0 & 1 & w & w^{2} & w & w & w & 1 & 0 & 0 & 0 & 0 \\
0 & 0 & 1 & w^{2} & w & w^{2} & w^{2} & w^{2} & 1 & 0 & 0 & 0 \\
0 & 0 & 0 & 1 & w & w^{2} & w & w & w & 1 & 0 & 0 \\
0 & 0 & 0 & 0 & 1 & w^{2} & w & w^{2} & w^{2} & w^{2} & 1 & 0 \\
0 & 0 & 0 & 0 & 0 & 1 & w & w^{2} & w & w & w & 1
\end{array}\right)
$$

We obtain a $[12,6,6]$ skew code over $\mathbb{F}_{4}$ of prescribed rank 2 which is a Gabidulin code. This code maps to a $[24,12,6]$ code over $\mathbb{F}_{2}$.

Example 4 Consider $\mathbb{F}_{q}=\mathbb{F}_{2^{4}}=\mathbb{F}_{16}, \theta(x)=x^{4}$ and $s=4$. This means, we will use elements $\beta \in \mathbb{F}_{2^{16}}$ in order to construct codes over $\mathbb{F}_{2^{4}}$. We denote by $\alpha$ the generator of $\mathbb{F}_{2^{16}}$ and $w$ the generator of $\mathbb{F}_{2^{4}}$ given by MAGMA.

1. Consider $\beta=\alpha^{57153}$. The longest sequence which is linearly independent over $\mathbb{F}_{2^{2}}=$ $\left(\mathbb{F}_{2^{4}}\right)^{\theta}$ is $\beta, \theta(\beta), \ldots, \theta^{7}(\beta)$. Therefore, if we obtain a skew code of constructed rank, this code must be of length $n=8$. Using the Casoratian determinant we compute $L_{\beta}(y)=\left(\theta^{8}-1\right)(y)$. Since the associated operator $f_{L}=X^{8}-1 \in \mathbb{F}_{2^{2}}[X, \theta]$ is a central polynomial (i.e. $f \in \mathbb{F}_{2^{2}}\left[X^{4}\right]$ ), we will be able to construct a skew code over $\mathbb{F}_{2^{4}}$ of constructed rank using this $\beta \in \mathbb{F}_{2^{16}}$. We start with $j=1$, and compute the smallest $\mathbb{F}_{2}$-space $V_{1}$ containing $\{\beta\}$ which is stable under the generator $\sigma: x \mapsto x^{16}$ of $\operatorname{Aut}\left(\mathbb{F}_{2^{16}} / \mathbb{F}_{16}\right)$. A basis of $V_{1}$ is $\left\{\beta, \theta^{2}(\beta), \theta^{4}(\beta), \theta^{6}(\beta)\right\}$ (note that $\sigma=\theta^{2}$ ). Using the Casarotian determinant, we compute the skew polynomial :

$$
\begin{aligned}
g & =X^{4}+\alpha^{17476} X^{3}+\alpha^{56797} X^{2}+\alpha^{39321} X+\alpha^{39321} \\
& =X^{4}+w^{4} X^{3}+w^{13} X^{2}+w^{9} X+w^{9}
\end{aligned}
$$

associated with the corresponding difference operator having the solution space $V_{1}$. Since the length of the skew code is 4 , the generator matrix of the code is :

$$
\left(\begin{array}{cccccccc}
w^{9} & w^{9} & w^{13} & w^{4} & 1 & 0 & 0 & 0 \\
0 & w^{6} & w^{6} & w^{7} & w & 1 & 0 & 0 \\
0 & 0 & w^{9} & w^{13} & w^{4} & 1 & 0 & 0 \\
0 & 0 & 0 & w^{6} & w^{6} & w^{7} & w & 1
\end{array}\right)
$$


We obtain a $[8,4,5]$ skew code over $\mathbb{F}_{16}$ of prescribed rank 2 which is a Gabidulin code. This code maps to a $[32,16,7]$ code over $\mathbb{F}_{2}$.

2. $\beta=\alpha^{18575}$. The longest sequence which is linearly independent over $\mathbb{F}_{2^{2}}=\left(\mathbb{F}_{2^{4}}\right)^{\theta}$ is $\beta, \ldots, \theta^{5}(\beta)$. Therefore, if we obtain a skew code of constructed rank, this code must be of length $n=6$. Using the Casoratian determinant we compute $L_{\beta}(y)=$ $\left(\theta^{6}+\theta^{4}+\theta^{2}+1\right)(y)$. Since the associated operator $f_{L}=X^{6}+X^{4}+X^{2}+1 \in \mathbb{F}_{2^{2}}[X, \theta]$ is a central polynomial (i.e. $f \in \mathbb{F}_{2^{2}}\left[X^{4}\right]$ ), we will be able to construct a skew code over $\mathbb{F}_{2^{4}}$ of constructed rank using this $\beta \in \mathbb{F}_{2^{16}}$. A basis of $V_{1}$ is $\left\{\beta, \theta^{2}(\beta), \theta^{4}(\beta)\right\}$ (note that $\left.\sigma=\theta^{2}\right)$. Using the Casarotian determinant, we compute the skew polynomial

$$
\begin{aligned}
g & =X^{3}+\alpha^{61166} X^{2}+\alpha^{56797} X+1 \\
& =X^{3}+w^{14} X^{2}+w^{13} X+1
\end{aligned}
$$

associated to the corresponding difference operator having the solution space $V_{1}$. Since the length of the skew code is 4 , the generator matrix of the code is :

$$
\left(\begin{array}{cccccc}
1 & w^{13} & w^{14} & 1 & 0 & 0 \\
0 & 1 & w^{7} & w^{11} & 1 & 0 \\
0 & 0 & 1 & w^{13} & w^{14} & 1
\end{array}\right)
$$

We obtain a $[6,3,4]$ skew code over $\mathbb{F}_{16}$ of prescribed rank 2 which is not a Gabidulin code.

The following table shows the characteristics of codes with prescribed rank that are defined over $\mathbb{F}_{4}$. The lines indicate the fields where $\beta$ has been found and the columns indicate the rank $\delta$ that has been prescribed during the construction (the actual prescribed distance could be larger). The entry $[10,5,4](8)$ means that 8 different skew polynomials $g$ have been found that lead to a $[10,5,4]$ code. We also indicate a Gabidulin code with an index $g$. Note that codes with the same properties can be obtained using elements $\beta$ in very different extensions. In the tables we only included those $\beta$ that do not belong to any subfield. In all the examples that follow, imposing a certain rank never results in a code having a higher rank. 


\begin{tabular}{|c||c|}
\hline & $\delta=1$ \\
\hline $\mathbb{F}_{4}$ & {$[\mathbf{2}, \mathbf{1}, \mathbf{2}]_{g}(2)$} \\
\hline \hline $\mathbb{F}_{4^{2}}$ & {$[\mathbf{4 , 2}, \mathbf{3}]_{g}(4)$} \\
\hline \hline \multirow{3}{*}{$\mathbb{F}_{4^{3}}$} & {$[6,3,2]_{g}(2)$} \\
& {$[6,3,3]_{g}(4)$} \\
& {$[\mathbf{6}, \mathbf{3}, \mathbf{4}]_{g}(2)$} \\
& {$[\mathbf{4}, \mathbf{2}, \mathbf{2}](4)$} \\
\hline \hline $\mathbb{F}_{4^{4}}$ & {$[\mathbf{8 , 4}, \mathbf{4}]_{g}(16)$} \\
& {$[\mathbf{6}, \mathbf{3}, \mathbf{3}](8)$} \\
\hline \hline & {$[10,5,2]_{g}(2)$} \\
& {$[10,5,4]_{g}(14)$} \\
$\mathbb{F}_{4^{5}}$ & {$[\mathbf{1 0 , 5}, \mathbf{5}]_{g}(16)$} \\
& {$[8,4,2](2)$} \\
& {$[8,4,3](2)$} \\
& {$[\mathbf{8}, \mathbf{4}, \mathbf{4}](12)$} \\
\hline
\end{tabular}

\begin{tabular}{|c||c|}
\hline & $\delta=1$ \\
\hline & {$[12,6,3]_{g}(12)$} \\
& {$[12,6,4]_{g}(12)$} \\
& {$[12,6,5]_{g}(32)$} \\
& {$[\mathbf{1 2}, \mathbf{6}, \mathbf{6}]_{g}(8)$} \\
$\mathbb{F}_{4^{6}}$ & {$[10,5,3]$} \\
& {$[\mathbf{1 0 , 5}, \mathbf{4}](8)$} \\
& {$[8,4,2](2)$} \\
& {$[8,4,3](8)$} \\
& {$[\mathbf{8}, \mathbf{4}, \mathbf{4}](22)$} \\
\hline
\end{tabular}

\begin{tabular}{|c||c|}
\hline \multicolumn{1}{|c||}{$\delta=1$} \\
\hline & {$[14,7,2]_{g}(2)$} \\
& {$[14,7,4]_{g}(14)$} \\
& {$[14,7,5]_{g}(40)$} \\
& {$[\mathbf{1 4}, \mathbf{7}, \mathbf{6}]_{g}(72)$} \\
& {$[12,6,2](2)$} \\
$\mathbb{F}_{4} 4^{7}$ & {$[12,6,4](30)$} \\
& {$[\mathbf{1 2}, \mathbf{6}, \mathbf{5}](32)$} \\
& {$[8,4,2](4)$} \\
& {$[8,4,3](24)$} \\
& {$[\mathbf{8}, \mathbf{4}, \mathbf{4}](4)$} \\
& {$[\mathbf{6}, \mathbf{3}, \mathbf{3}](16)$} \\
\hline
\end{tabular}

The bold entries correspond to the best possible minimum distance with fixed length and dimension. The following tables, organized in the same way as the previous tables, show the characteristics of codes with prescribed rank that are defined over $\mathbb{F}_{8}$.

\begin{tabular}{|c|c|c|}
\hline & $\delta=1$ & $\delta=2$ \\
\hline$\overline{F_{8}}$ & {$[\mathbf{3}, \mathbf{2}, 2]_{g}(3)$} & $\overline{[3, \mathbf{1}, \mathbf{3}]_{g}(3)}$ \\
\hline \multirow[t]{2}{*}{$\overline{\mathbb{F}_{8^{2}}}$} & $\overline{[6,4,2]_{g}(3)}$ & $\overline{[6,2,3]_{g}(3)}$ \\
\hline & {$[\mathbf{6}, \mathbf{4}, \mathbf{3}]_{g}(9)$} & {$[\mathbf{6}, \mathbf{2}, \mathbf{5}]_{g}(9)$} \\
\hline \multirow{3}{*}{$\mathbb{F}_{8^{3}}$} & {$[9,6,3]_{g}(54)$} & {$[9,3,6]_{g}(54)$} \\
\hline & {$[\mathbf{9}, \mathbf{6}, \mathbf{4}]_{g}(9)$} & {$[\mathbf{9}, \mathbf{3}, \mathbf{7}]_{g}(9)$} \\
\hline & {$[\mathbf{6}, \mathbf{4}, \mathbf{3}](21)$} & {$[\mathbf{6}, \mathbf{2}, \mathbf{5}](21)$} \\
\hline \multirow{8}{*}{$\mathbb{F}_{8^{4}}$} & {$[12,8,2]_{g}(3)$} & {$[12,4,3]_{g}(3)$} \\
\hline & {$[12,8,3]_{g}(63)$} & {$[12,4,5]_{g}(9)$} \\
\hline & {$[\mathbf{1 2}, \mathbf{8}, \mathbf{4}]_{g}(126)$} & {$[12,4,6]_{g}(54)$} \\
\hline & {$[9,6,2](3)$} & {$[12,4,7]_{g}(54)$} \\
\hline & {$[\mathbf{9}, \mathbf{6}, \mathbf{3}](45)$} & $12,4,8]_{g}(72)$ \\
\hline & & {$[9,3,3](3)$} \\
\hline & & {$[9,3,5](9)$} \\
\hline & & {$[\mathbf{9}, \mathbf{3}, \mathbf{6}](36)$} \\
\hline
\end{tabular}

\begin{tabular}{|c|c|c|}
\hline & $\delta=1$ & $\delta=2$ \\
\hline \multirow{9}{*}{$\mathbb{F}_{8^{5}}$} & {$[15,10,2]_{g}(5)$} & {$[15,5,3]_{g}$} \\
\hline & {$[15,10,3]_{g}(120)$} & {$[15,5,6]_{g}$} \\
\hline & {$[15,10,4]_{g}(432)$} & {$[15,5,7]_{g}$} \\
\hline & {$[\mathbf{1 5}, \mathbf{1 0}, \mathbf{5}]_{g}(120)$} & {$[15,5,8]_{g}$} \\
\hline & {$[12,8,2](3)$} & {$[15,5,9]_{g}$} \\
\hline & {$[12,8,3](78)$} & {$[12,4,3]^{\top}$} \\
\hline & {$[\mathbf{1 2}, \mathbf{8}, \mathbf{4}](144)$} & {$[12,4,5]$} \\
\hline & & {$[12,4,6]$} \\
\hline & & {$[12,4,7]$} \\
\hline
\end{tabular}

The following tables, organized in the same way as the previous tables, show the characteristics of codes with prescribed rank that are defined over $\mathbb{F}_{16}$. 


\begin{tabular}{|c|c|c|c|}
\hline & $\delta=1$ & $\delta=2$ & $\delta=3$ \\
\hline $\mathbb{F}_{16}$ & {$[4,3,2]_{g}(8)$} & {$[4,2,3]_{g}(8)$} & {$[4,1,4]_{g}(8)$} \\
\hline $\mathbb{F}_{16^{2}}$ & {$[\mathbf{8}, \mathbf{6}, \mathbf{3}]_{g}(64)$} & $\begin{array}{l}{[8,4,4]_{g}(32)} \\
{[\mathbf{8}, \mathbf{4}, \mathbf{5}]_{g}(32)}\end{array}$ & {$[\mathbf{8}, \mathbf{2}, \mathbf{7}]_{g}(64)$} \\
\hline $\mathbb{F}_{16^{3}}$ & $\begin{array}{c}{[12,9,2]_{g}(32)} \\
{[12,9,3]_{g}(408)} \\
{[\mathbf{1 2}, \mathbf{9}, \mathbf{4}]_{g}(72)} \\
{[8,6,2](16)} \\
{[\mathbf{8}, \mathbf{6}, \mathbf{3}](48)}\end{array}$ & $\begin{array}{c}{[12,6,3]_{g}(8)} \\
{[12,6,4]_{g}(72)} \\
{[12,6,5]_{g}(136)} \\
{[\mathbf{1 2}, \mathbf{6}, \mathbf{6}]_{g}(296)} \\
{[8,4,3](16)} \\
{[8,4,4](40)} \\
{[\mathbf{8}, \mathbf{4}, \mathbf{5}](8)}\end{array}$ & $\begin{array}{c}{[12,3,4]_{g}(8)} \\
{[12,3,6]_{g}(64)} \\
{[12,3,8]_{g}(152)} \\
{[12,3,9]_{g}(216)} \\
{[\mathbf{1 2}, \mathbf{3}, \mathbf{1 0}]_{g}(72)} \\
{[8,2,4](8)} \\
{[8,2,5](8)} \\
{[\mathbf{8}, \mathbf{2}, \mathbf{7}](48)}\end{array}$ \\
\hline $\mathbb{F}_{16^{4}}$ & $\begin{array}{c}{[16,12,3]_{g}(256)} \\
{[\mathbf{1 6}, \mathbf{1 2}, \mathbf{4}]_{g}(3840)} \\
{[\mathbf{1 2}, \mathbf{9}, \mathbf{3}](512)}\end{array}$ & $\begin{array}{c}{[16,8,6]_{g}(368)} \\
{[16,8,7]_{g}(3008)} \\
{[\mathbf{1 6}, \mathbf{8}, \mathbf{8}]_{g}(720)} \\
{[12,6,5]_{(192)}} \\
{[\mathbf{1 2}, \mathbf{6}, \mathbf{6}](320)}\end{array}$ & $\begin{array}{c}{[16,4,10]_{g}(256)} \\
{[16,4,11]_{g}(1536)} \\
{[\mathbf{1 6}, \mathbf{4}, \mathbf{1 2}]_{g}(2304)} \\
{[\mathbf{1 2}, \mathbf{3}, \mathbf{8}](512)}\end{array}$ \\
\hline
\end{tabular}

Example 5 Using elements in $\mathbb{F}_{88}$, we found the following codes $[21,14,6]$ (best known distance) defined over $\mathbb{F}_{8}$ :

1. The code generated by

$$
g=X^{7}+w X^{6}+w^{3} X^{5}+w^{5} X^{4}+w^{6} X^{3}+w^{4} X^{2}+w \in \mathbb{F}_{8}[X, \theta]
$$

where $w$ is the generator of $\mathbb{F}_{8}$ given by MAGMA. The bound of $g$ is $f=X^{21}+X^{18}+$ $X^{15}+X^{12}+X^{9}+X^{6}+X^{3}+1 \in \mathbb{F}_{2}\left[X^{3}\right]$. This code is not a Gabidulin code.

2. The code generated by

$$
g=X^{7}+w X^{6}+w^{3} X^{5}+w^{4} X^{4}+w^{5} X^{3}+w^{3} X^{2}+w X+w^{2} \in \mathbb{F}_{8}[X, \theta]
$$

where $w$ the generator of $\mathbb{F}_{8}$ given by Magma. The bound of $g$ is $f=X^{21}+1 \in$ $\mathbb{F}_{2}\left[X^{3}\right]$. According to its bound, this code is a Gabidulin code.

Example 6 Consider $\mathbb{F}_{q}=\mathbb{F}_{8}, \theta(x)=x^{2}$ and $s=14$. This means that we will use elements in $\mathbb{F}_{8^{14}}$ in order to construct codes over $\mathbb{F}_{8}$. We denote by $\alpha$ the generator of $\mathbb{F}_{2^{42}}$ and $w$ the generator of $\mathbb{F}_{8}$ given by MAGMA. Consider $\beta=\alpha^{70193}$. The longest sequence which is linearly independent over $\mathbb{F}_{2}=\left(\mathbb{F}_{8}\right)^{\theta}$ is $\beta, \ldots, \theta^{41}(\beta)$. Using the Casoratien determinant we compute $L_{\beta}(y)=\theta^{42}(y)+y$. The corresponding skew polynomial $f=$ $X^{42}+1$ is central. We set $\delta=2$. The smallest $\mathbb{F}_{2}$-space containing $\{\beta, \theta(\beta)\}$ which is stable under $\sigma(x)=x^{8}$ has dimension 28 and the skew polynomial $g$ is :

$$
g=X^{28}+w^{2} X^{27}+X^{26}+w^{5} X^{25}+w^{3} X^{24}+w X^{23}+w^{2} X^{22}+w^{4} X^{21}
$$




$$
\begin{gathered}
+w^{2} X^{19}+X^{18}+w^{5} X^{17}+w^{4} X^{16}+w X^{15}+X^{14}+w^{2} X^{13}+w^{4} X^{12}+w^{4} X^{11} \\
+w^{4} X^{10}+w^{5} X^{9}+w^{5} X^{7}+w^{6} X^{6}+w^{5} X^{5}+w^{5} X^{4}+w^{6} X^{3}+w^{4} X^{2}+w^{6} X+w
\end{gathered}
$$

We can compute as usual the generator matrix and we obtain a $\theta$-cyclic code over $\mathbb{F}_{8}$ with parameters $[42,14,21]$. This code improves by one the minimum distance of the previous best known linear code of length 42 and dimension 14 (according to [10]). The upper bound of the minimum distance (here the Griesmer bound) is , in this case, 25.

\section{Skew codes with constructed distance}

In the previous section we worked with solutions of linear difference equations, while in this section we will consider (right) roots of the associated skew polynomial.

Definition $4 \alpha \in \mathbb{F}_{q^{s}}$ is a root of the skew polynomial $f \in \mathbb{F}_{q}[X, \theta]$ if and only if $f$ is right divisible by $X-\alpha$.

These codes are built in analogy to $\mathrm{BCH}$ codes and in the next section we adapt the classical algorithm for $\mathrm{BCH}$ codes to decode them.

As for linear difference equations, it is possible to find the roots of a skew polynomial by solving an associated commutative polynomial. Following [11], for $\alpha \in \mathbb{F}_{q^{s}}$ and $\theta \in$ $\operatorname{Aut}\left(\mathbb{F}_{q^{s}}\right)$ we denote $\mathcal{N}_{\theta, i}(\alpha)=\theta^{i-1}(\alpha) \cdots \theta(\alpha) \cdot \alpha$. From [11] Theorem 1.3.11 we get that $a_{n} X^{n}+\ldots+a_{1} X+a_{0} \in \mathbb{F}_{q}[X, \theta]$ is right divisible by $X-\alpha$ if and only if $\alpha$ is a zero of the associated polynomial

$$
\mathcal{P}_{f}=\sum_{i=0}^{n} a_{i} \mathcal{N}_{\theta, n-i}(Y) \in \mathbb{F}_{q}[Y] .
$$

If $\theta$ is defined by $a \mapsto a^{q_{0}}$, this corresponds to

$$
\begin{aligned}
\mathcal{P}_{f} & =\sum_{i=0}^{n} a_{i} Y^{q_{0}^{i-1}+q_{0}^{i-2}+\ldots+1} \\
& =\sum_{i=0}^{n} a_{i} Y^{\frac{q_{0}^{i}-1}{q_{0}-1}} \in \mathbb{F}_{q}[Y]
\end{aligned}
$$

The following Lemma shows the link between roots of skew polynomials and solutions of the associated difference equation :

Lemma 4 Let $\theta$ be an automorphism of $\mathbb{F}_{q}, L(y)=\sum_{i=0}^{n} a_{i} \theta^{i}(y)$ a difference equation and $P=\sum_{i=0}^{n} a_{i} X^{i}$ the corresponding operator in $\mathbb{F}_{q}[X, \theta]$. Then a non zero element $\beta$ of $\mathbb{F}_{q^{s}}$ is a solution of $L(y)=0$ if and only if $X-\frac{\theta(\beta)}{\beta}$ is a right divisor of $P_{L}$ in $\mathbb{F}_{q^{s}}[X, \theta]$. 
Proof. Suppose that $L(\beta)=0$. Using the right euclidean algorithm we obtain $P_{L}=Q \cdot\left(X-\frac{\theta(\beta)}{\beta}\right)+R$ where $R \in \mathbb{F}_{q}$. Since $\beta$ is a solution of $\left(\theta-\frac{\theta(\beta)}{\beta}\right)(y)=0$, we obtain $0=L(\beta)=P_{L}(\beta)=R \cdot \beta$, showing that $R=0$. Conversely if $R=0$ then $X-\frac{\theta(\alpha)}{\alpha}$ is a right factor of $P_{L}$, showing that $L(\alpha)=0$.

Lemma 5 Let $\theta \in \operatorname{Aut}\left(\mathbb{F}_{q}\right)$ be defined by $a \mapsto a^{q_{0}}$ and $\mathbb{F}_{q_{0}}=\left(\mathbb{F}_{q}\right)^{\theta}$ be the fixed field of $\theta$ in $\mathbb{F}_{q}$. If $\mathbb{F}_{q} \subset \mathbb{F}_{q^{s}}$ is an extension of finite fields and $\theta \in A u t\left(\mathbb{F}_{q} /\left(\mathbb{F}_{q}\right)^{\theta}\right)$, then for any $\sigma$ in Aut $\left(\mathbb{F}_{q^{s}} / \mathbb{F}_{q}\right)$ the map

$$
\begin{aligned}
\varphi_{\sigma}: \mathbb{F}_{q^{s}}[X, \theta] & \rightarrow \mathbb{F}_{q^{s}}[X, \theta] \\
\sum_{i=0}^{n} a_{i} X^{i} & \mapsto \sum_{i=0}^{n} \sigma\left(a_{i}\right) X^{i}
\end{aligned}
$$

is a morphism of rings.

Proof. We denote again $\theta$ the extension of the automorphism

$$
\begin{aligned}
\theta: \mathbb{F}_{q} & \rightarrow \mathbb{F}_{q} \\
a & \mapsto a^{q_{0}}
\end{aligned}
$$

to $\mathbb{F}_{q^{s}}$. Since the addition in $\mathbb{F}_{q^{s}}[X, \theta]$ is the same as in $\mathbb{F}_{q^{s}}[X]$ and since $\sigma: \mathbb{F}_{q^{s}} \rightarrow \mathbb{F}_{q^{s}}$ can be extended to a morphism of $\mathbb{F}_{q^{s}}[X]$ by $X \mapsto X$, we get that $\varphi_{\sigma}$ is a morphism of the additive group $\left(\mathbb{F}_{q^{s}}[X, \theta],+\right)$. For the mutiplication we have $\varphi_{\sigma}(a X)=\varphi_{\sigma}(a) \varphi_{\sigma}(X)$, but we also need $\varphi_{\sigma}(X a)=\varphi_{\sigma}(X) \varphi_{\sigma}(a)$, or equivalently

$$
\sigma(\theta(a)) X=\varphi_{\sigma}(\theta(a) X)=\varphi_{\sigma}(X a)=\varphi_{\sigma}(X) \varphi_{\sigma}(a)=\theta(\sigma(a)) X .
$$

The condition turns out to be $\theta \sigma=\sigma \theta$. The later is true because $\operatorname{Aut}\left(\mathbb{F}_{\mathrm{q}^{\mathrm{s}}} /\left(\mathbb{F}_{\mathrm{q}^{\mathrm{s}}}\right)^{\theta}\right)$ is cyclic and $\sigma$ is a power of the generator of $\operatorname{Aut}\left(\mathbb{F}_{\mathrm{q}^{\mathrm{s}}} /\left(\mathbb{F}_{\mathrm{q}^{\mathrm{s}}}\right)^{\theta}\right)$.

Lemma 6 Be $f=a_{n} X^{n}+\ldots+a_{1} X+a_{0} \in \mathbb{F}_{q}[X, \theta]$ and assume that $a_{0} \neq 0$, then there is a finite field extension $\mathbb{F}_{q^{s}}$ such that $f$ is the least common left multiple of the polynomials $X-\alpha_{i}$, where $\alpha_{i} \in \mathbb{F}_{q^{s}}$.

Proof. Since $a_{0} \neq 0$, Theorem 1 shows that a finite splitting field exists for $L_{f}(y)=0$. For any solutions $\beta \in \mathbb{F}_{q^{s}}$ of $L_{f}(y)=0$, we have that $X-\frac{\theta(\beta)}{\beta}$ is a right factor of $f$ in $\mathbb{F}_{q^{s}}[X, \theta]$. Therefore the least common left multiple $g$ of the $X-\frac{\theta(\beta)}{\beta}$, where $\beta$ runs over all the solutions of $L_{f}(y)=0$ is also a right factor of $f$. Let $\sigma$ be a generator of $\operatorname{Aut}\left(\mathbb{F}_{q^{s}} / \mathbb{F}_{q}\right)$, then $\sigma$ commutes with the extension of $\theta$ to $\mathbb{F}_{q^{s}}$ and therefore maps a solution of $L_{f}(y)=0$ into another solution. This shows that $\varphi_{\sigma}$ maps $g$ to itself and therefore $g$ is defined over $\mathbb{F}_{q}$. Since all solutions of $f$ are solutions of $g$, the degree of $g$ must be the same as the degree of $f$, which show that they coincide.

In analogy to $\mathrm{BCH}$ codes, skew $\mathrm{BCH}$ codes with designed distance are introduced in [5] in the case where $q$ is a power of 2 . We now extend this definition to an arbitrary field $\mathbb{F}_{q}$ : 
Definition 5 Suppose that $\theta \in A u t\left(\mathbb{F}_{q}\right)$ is defined by $a \mapsto a^{q_{0}}$ and that $q=q_{0}^{t}$. A skew $B C H$ code of length $n$ over $\mathbb{F}_{q}$ for the non zero positive integer parameters $\delta$ and $s$ is a $\theta$-code that is generated by a skew polynomial $g \in \mathbb{F}_{q}[X, \theta]$ with the property that

1. $g \in \mathbb{F}_{q}[X, \theta]$ is the skew polynomial of smallest degree that is right divisible by $X-\alpha^{k}$ for $k \in\{1, \ldots, \delta-1\}$ where $\alpha$ is a generator of the multiplicative group of $\mathbb{F}_{q_{0}^{s}}$.

2. $g$ is bounded by a polynomial $f$ of degree $n$ (i.e. $f=h \cdot g$ and $(f) \subset \mathbb{F}_{q}[X, \theta]$ is a two-sided ideal).

We note such a code a $\left(n, q_{0}, t, s, \delta\right)$ skew $B C H$ code.

The following is a generalization of ([5], Proposition 2):

Proposition 2 If $n \leq\left(q_{0}-1\right) \cdot s$, then a $\left(n, q_{0}, t, s, \delta\right)$ skew $B C H$ code has minimum distance at least $\delta$.

Proof. Suppose that the code is generated by $g \in \mathbb{F}_{q}[X, \theta]$ and that $f$ is a bound of degree $n$ of $g$. An element $h=\sum_{i=0}^{n-1} c_{i} X^{i} \in \mathbb{F}_{q}[X, \theta] /(f)$ is a codeword if and only if it is a left multiple of $g$, or equivalently, if $\alpha^{k}$ is a root of $\mathcal{P}_{h}(Y) \in \mathbb{F}_{q}[Y]$ for $k \in\{1, \ldots, \delta-1\}$. We write $[i]=\frac{q_{0}^{i}-1}{q_{0}-1}$ and like in the proof of ([5], Proposition 2) we obtain a parity-check matrix of the form :

$$
\left(\begin{array}{cccccc}
\alpha^{[0]} & \alpha^{[1]} & \cdots & \alpha^{[\delta-1]} & \cdots & \alpha^{[n-1]} \\
\left(\alpha^{2}\right)^{[0]} & \left(\alpha^{2}\right)^{[1]} & \cdots & \left(\alpha^{2}\right)^{[\delta-1]} & \cdots & \left(\alpha^{2}\right)^{[n-1]} \\
\vdots & & & & & \vdots \\
\left(\alpha^{\delta-1}\right)^{[0]} & \left(\alpha^{\delta-1}\right)^{[1]} & \cdots & \left(\alpha^{\delta-1}\right)^{[\delta-1]} & \cdots & \left(\alpha^{\delta-1}\right)^{[n-1]}
\end{array}\right) .
$$

The determinant of all extracted $(\delta-1) \times(\delta-1)$ is non zero if and only if $\alpha^{[i]}-\alpha^{[j]} \neq 0$ for $i>j$ in $\{0,1, \ldots, n-1\}$. This follows from $\alpha^{[i]}=\alpha^{[j]}$ if and only if :

$$
\alpha^{\frac{q_{0}^{i}-q_{0}^{j}}{q_{0}-1}}=\alpha^{\frac{q_{0}^{j} \cdot\left(q_{0}^{i-j}-1\right)}{q_{0}-1}}=1 .
$$

In particular $\alpha^{q_{0}^{j} \cdot} \cdot\left(q_{0}^{i-j}-1\right)=1$, which implies that $q_{0}^{i-j}-1$ is divisible by $q_{0}^{s}-1$, the order of $\alpha$. This shows that $i-j=m \cdot s$ and from relation (3), we now get that $q_{0}-1$ must divide

$$
\frac{q_{0}^{m \cdot s}-1}{q_{0}^{s}-1}=\sum_{r=0}^{m-1} q_{0}^{s \cdot k} .
$$

Therefore $q_{0}-1$ divides $m$, showing that $i-j$ is a multiple of $\left(q_{0}-1\right) \cdot s$. Since $i-j<n \leq\left(q_{0}-1\right) \cdot s$, this is impossible.

Denote $\alpha$ a generator of the multiplicative group of $\mathbb{F}_{q_{0}^{s}}$. Note that $g$ is right divisible by $X-\alpha^{i}$, if and only if the solution $\beta_{i}$ of $\theta(y)-\alpha^{i} y=0$, is a solution of the difference equation $L_{g}(y)=0$ associated to $g$, i.e. $\theta\left(\beta_{i}\right) / \beta_{i}=\alpha^{i}$. The fact that $g \in \mathbb{F}_{q}[X, \theta]$ is the 
skew polynomial of smallest degree that is right divisible by $X-\alpha^{k}$ for $k \in\{1, \ldots, \delta-1\}$ is equivalent to the fact that the solution space $V_{g}$ of $L_{g}(y)=0$ contains $\beta_{1}, \beta_{2}, \ldots, \beta_{\delta-1}$. According to Lemma 2, for $L_{g}(y)$ (and therefore $g$ ) to be defined over $\mathbb{F}_{q}$, the vector space $V_{g}$ must be stable under a generator $\sigma$ of $\operatorname{Aut}\left(\mathbb{F}_{q_{0}^{s}} / \mathbb{F}_{q}\right)$. The following gives an algorithm to find the $\left(n, q_{0}, t, s, \delta\right)$ skew $\mathrm{BCH}$ codes with designed distance $\delta \geq \Delta$ :

1. For each generator $\alpha$ of the multiplicative group of $\mathbb{F}_{q_{0}^{s}}$, compute $\beta_{i}$ (where $i \in$ $\{1, \ldots, \Delta-1\})$ such that $\theta\left(\beta_{i}\right) / \beta_{i}=\alpha^{i}$.

2. Compute the smallest $\mathbb{F}_{q_{0}}$-vector space $V_{g}$ that contains $\beta_{1}, \beta_{2}, \ldots, \beta_{\Delta-1}$ which is invariant under a generator $\sigma$ of $\operatorname{Aut}\left(\mathbb{F}_{q_{0}^{s}} / \mathbb{F}_{q}\right)$.

3. Using the Casarotian determinant on a basis of $V_{g}$, compute the skew polynomial $g \in \mathbb{F}_{q}[X, \theta]$ associated to the corresponding difference operator, having the solution space $V_{g}$.

4. Compute a bound $f \in \mathbb{F}_{q_{0}}\left[X^{m}\right]$ for $g$ (here $m$ denotes the order of $\theta \in \operatorname{Aut}\left(\mathbb{F}_{q}\right)$ ). If the degree $n$ of $f$ is such that $n \leq\left(q_{0}-1\right) \cdot s$, then $g$ will denote a skew BCH code of designed distance $\geq \Delta$.

5. In order to compute the real designed distance of the resulting code, we compute the largest integer $\delta$ with the property, that $\beta_{1}, \beta_{2}, \ldots, \beta_{\delta-1}$ are solutions of $L_{g}(y)=0$.

In order to simplify the computation of the bound $f$ of $g$, we can use the fact that the solution space of $f \in \mathbb{F}_{q_{0}}[X, \delta] \subset \mathbb{F}_{q_{0}}\left[X^{m}\right]$ must be invariant under $\Theta \in \operatorname{Aut}\left(\mathbb{F}_{q_{0} s} / \mathbb{F}_{q_{0}}\right)$ (Lemma 2). In particular the smallest $\mathbb{F}_{q_{0}}$-vector space $V_{F}$, that contains $V_{g}$ and is invariant under $\Theta \in \operatorname{Aut}\left(\mathbb{F}_{q_{0} s} / \mathbb{F}_{q_{0}}\right)$, will be included in the solution space $V_{f}$ of $L_{f}(y)=0$. If we denote $F \in \mathbb{F}_{q_{0}}[X, \theta]=\mathbb{F}_{q_{0}}[X]$ the skew polynomial associated to the difference equation whose solution space is $V_{F}$, then $f$ is (right) divisible by $F$ in $\mathbb{F}_{q_{0}}[X, \theta]=\mathbb{F}_{q_{0}}[X]$. The computation of the bound $f$ in the above procedure can therefore be replaced by :

1. Compute the smallest $\mathbb{F}_{q_{0}}$-vector space $V_{F}$ that contains $V_{g}$ and is invariant under $\Theta \in \operatorname{Aut}\left(\mathbb{F}_{q_{0} s} / \mathbb{F}_{q_{0}}\right)$.

2. Compute the skew polynomial $F \in \mathbb{F}_{q_{0}}[X, \theta]=\mathbb{F}_{q_{0}}[X]$ associated to the corresponding difference operator having the solution space $V_{F}$.

3. If the degree of $F$ is $\tau$, then consider a polynomial $h \in \mathbb{F}_{q_{0}}[X]$ with unknown coefficients of degree $\left(q_{0}-1\right) \cdot s-\tau$. Verify if there are values in $\mathbb{F}_{q_{0}}$ for the unknown coefficients so that $h \cdot F$ belongs to $\mathbb{F}_{q_{0}}\left[X^{m}\right]$ for $g$ (here $m$ denotes the order of $\left.\theta \in \operatorname{Aut}\left(\mathbb{F}_{q}\right)\right)$. This leads to a system of linear equations over $\mathbb{F}_{q_{0}}$, which can be obtained by setting the corresponding coefficients of $h \cdot F$ to zero.

Example 7 Consider $\mathbb{F}_{q}=\mathbb{F}_{2^{3}}, \theta: x \mapsto x^{2}$ and $s=3$. This means that we will use elements $\alpha \in \mathbb{F}_{2^{9}}$ in order to construct codes over $\mathbb{F}_{2^{3}}$. We denote by $\gamma$ the generator of $\mathbb{F}_{2^{9}}$ and 
by $w$ the generator of $\mathbb{F}_{2^{3}}$ given by MAGMA. Consider $\alpha=\gamma^{433}$ and $\delta=2$. The smallest $\mathbb{F}_{2}=\left(\mathbb{F}_{2^{3}}\right)^{\theta}$-space $V_{\alpha, \alpha^{2}}$ containing $\left\{\alpha, \alpha^{2}\right\}$ which is stable under the generator $\sigma: x \mapsto x^{8}$ of Aut $\left(\mathbb{F}_{2^{9}} / \mathbb{F}_{3}\right)$ has a basis $\left\{\alpha, \gamma^{483}, \gamma^{410}, \gamma^{179}\right\}$. Using the Casarotian determinant, we compute :

$$
L_{\alpha, \gamma^{483}, \gamma^{410}, \gamma^{179}}(y)=\theta^{4}(y)+w^{2} \theta^{3}(y)+w \theta^{2}(y)+w \theta(y)+y
$$

The skew polynomial $g=X^{4}+w^{2} X^{3}+w X^{2}+w X+1 \in \mathbb{F}_{2^{3}}[X, \theta]$ will be the generator of the skew code we are constructing. The bound of $g$ is $f=X^{6}+X^{3}+1 \in \mathbb{F}_{2}\left[X^{3}\right]$. Therefore the length of the skew code will be 6 and the generator matrix of the code is :

$$
\left(\begin{array}{cccccc}
1 & w & w & w^{2} & 1 & 0 \\
0 & 1 & w^{2} & w^{2} & w^{4} & 1
\end{array}\right)
$$

We obtain a $[6,2,5]$ skew code over $\mathbb{F}_{8}$ of prescribed distance 3 .

The following tables show the characteristics of codes with prescribed distance that are defined over $\mathbb{F}_{4}$. The lines indicate the fields where $\alpha$ has been found and the columns indicate the distance $\Delta$ prescribed during the construction (the actual prescribed distance could be larger). The entry $[6,3,3](8)$ means that 8 different skew polynomials $g$ have been found for the values $[6,3,3]$. In the following table we can observe that prescribing a certain distance may result in a code with the same characteristics where a higher distance could have been prescribed. 


\begin{tabular}{|c|c|c|c|c|c|c|}
\hline & $\Delta=2$ & $\Delta=3$ & $\Delta=4$ & $\Delta=5$ & $\Delta=6$ & $\Delta=7$ \\
\hline $\mathbb{F}_{2^{6}}$ & $\begin{array}{l}{[6,3,3](6)} \\
{[6,3,4](6)}\end{array}$ & $\begin{array}{l}{[6,1,6](1)} \\
{[6,2,4](1)}\end{array}$ & {$[6,1,6](1)$} & {$[6,1,6](1)$} & & \\
\hline $\mathbb{F}_{2^{8}}$ & $\begin{array}{c}{[8,4,4](20)} \\
{[6,3,3](8)}\end{array}$ & $\begin{array}{l}{[8,1,8](1)} \\
{[6,1,4](1)}\end{array}$ & {$[8,1,8](3)$} & & & \\
\hline $\mathbb{F}_{2^{10}}$ & $\begin{array}{l}{[10,5,4](24)} \\
{[10,5,5](24)} \\
{[\mathbf{1 0}, \mathbf{6}, \mathbf{3}](2)} \\
[\mathbf{8}, \mathbf{4}, \mathbf{4}](12))\end{array}$ & $\begin{array}{c}10,1,10](1) \\
{[10,4,4](1)}\end{array}$ & {$[10,1,10](3)$} & {$[10,1,10](3)$} & {$[10,1,10](3)$} & {$[10,1,10](3)$} \\
\hline $\mathbb{F}_{2^{12}}$ & $\begin{array}{c}{[12,6,3](12)} \\
{[12,6,4](18)} \\
{[12,6,5](54)} \\
{[\mathbf{1 2}, \mathbf{6}, \mathbf{6}](12)} \\
{[12,7,3](6)} \\
{[\mathbf{1 2}, \mathbf{7}, \mathbf{4}](12)} \\
{[\mathbf{1 2}, \mathbf{8}, \mathbf{3}](6)} \\
{[10,5,3](6)} \\
{[\mathbf{1 0}, \mathbf{5}, \mathbf{4}](18)} \\
{[8,4,3](12)} \\
{[\mathbf{8}, \mathbf{4}, \mathbf{4}](18)}\end{array}$ & $\begin{array}{c}{[\mathbf{1 2}, \mathbf{1}, \mathbf{1 2}](1)} \\
{[\mathbf{1 2}, \mathbf{2}, \mathbf{8}](1)} \\
{[\mathbf{1 2}, \mathbf{3}, \mathbf{4}](1)} \\
{[\mathbf{1 0}, \mathbf{1}, \mathbf{6}](1)} \\
{[10,2,4](1)} \\
{[\mathbf{1 0}, \mathbf{3}, \mathbf{4}](1)} \\
{[8,1,4](1)} \\
{[8,2,4](1)} \\
{[8,2,5](1)} \\
{[8,3,4](1)}\end{array}$ & $\begin{array}{c}{[\mathbf{1 2}, \mathbf{1}, \mathbf{1 2}](3)} \\
{[12,2,6](2)} \\
{[12,2,8](3)} \\
{[\mathbf{1 2}, \mathbf{2}, \mathbf{9}](4)} \\
{[\mathbf{1 2}, \mathbf{4}, \mathbf{6}](2)}\end{array}$ & $\begin{array}{c}{[\mathbf{1 2}, \mathbf{1}, \mathbf{1 2}](3)} \\
{[12,2,6](2)} \\
{[12,2,8](3)} \\
{[\mathbf{1 2}, \mathbf{2}, \mathbf{9}](3)} \\
{[\mathbf{1 2}, \mathbf{4}, \mathbf{6}](2)}\end{array}$ & $\begin{array}{c}{[\mathbf{1 2}, \mathbf{1}, \mathbf{1 2}](3)} \\
{[12,2,8](1)} \\
{[\mathbf{1 2}, \mathbf{2}, \mathbf{9}](4)}\end{array}$ & $\begin{array}{c}{[\mathbf{1 2}, \mathbf{1}, \mathbf{1 2}](3)} \\
{[12, \mathbf{2}, \mathbf{8}](1)}\end{array}$ \\
\hline $\mathbb{F}_{2^{14}}$ & $\begin{array}{c}{[14,7,4](24)} \\
{[14,7,5](84)} \\
{[\mathbf{1 4}, \mathbf{7}, \mathbf{6}](132)} \\
{[12,6,4](30)} \\
{[\mathbf{1 2}, \mathbf{6}, \mathbf{5}](48)} \\
{[8,4,3](24)} \\
{[\mathbf{8}, \mathbf{4}, \mathbf{4}](8)} \\
{[\mathbf{6}, \mathbf{3}, \mathbf{3}](8)}\end{array}$ & $\begin{array}{c}14,1,14](1) \\
{[14,3,8](2)} \\
{[14,4,6](2)} \\
{[14,6,4](1)}\end{array}$ & $\begin{array}{c}{[\mathbf{1 4}, \mathbf{1}, \mathbf{1 4}](3)} \\
{[14,3,8](2)} \\
{[\mathbf{1 4}, \mathbf{3}, \mathbf{1 0}](4)} \\
{[14,4,6](2)} \\
{[\mathbf{1 4}, \mathbf{4}, \mathbf{8}](4)}\end{array}$ & $\begin{array}{c}{[\mathbf{1 4}, \mathbf{1}, \mathbf{1 4}](3)} \\
{[14,3,8](2)} \\
{[\mathbf{1 4}, \mathbf{3}, \mathbf{1 0}](4)} \\
{[\mathbf{1 4}, \mathbf{4}, \mathbf{6}](2)}\end{array}$ & $\begin{array}{c}{[14,1,14](3)} \\
{[14,3,8](1)}\end{array}$ & $\begin{array}{c}{[14,1,14](3)} \\
{[14,3,8](1)}\end{array}$ \\
\hline
\end{tabular}

The following table, organized in the same way as the previous table, shows the characteristics of codes with prescribed distances that are defined over $\mathbb{F}_{8}$. 


\begin{tabular}{|c|c|c|c|c|}
\hline & $\Delta=2$ & $\Delta=3$ & $\Delta=4$ & $\Delta=5$ \\
\hline \multirow[t]{2}{*}{$\mathbb{F}_{8^{2}}$} & {$[\mathbf{6}, \mathbf{4}, \mathbf{3}](18)$} & {$[\mathbf{6}, \mathbf{2}, \mathbf{5}](12)$} & {$[\mathbf{6}, \mathbf{2}, \mathbf{5}](9)$} & {$[\mathbf{6}, \mathbf{1}, \mathbf{6}](3)$} \\
\hline & & {$[6, \mathbf{6}, \mathbf{4}](3)$} & {$[\mathbf{6}, \mathbf{1}, \mathbf{6}](3)$} & \\
\hline \multirow{6}{*}{$\mathbb{F}_{8^{3}}$} & {$[9,6,3](108)$} & {$[9,3,6](60)$} & {$[\mathbf{9}, \mathbf{1}, \mathbf{9}](6)$} & {$[\mathbf{9}, \mathbf{1}, \mathbf{9}](3)$} \\
\hline & {$[\mathbf{9}, \mathbf{6}, \mathbf{4}](18)$} & {$[\mathbf{9}, \mathbf{3}, \mathbf{7}](12)$} & {$[9,2,6](6)$} & {$[\mathbf{9}, \mathbf{2}, \mathbf{6}](6)$} \\
\hline & {$[\mathbf{6}, \mathbf{3}, \mathbf{4}](18)$} & {$[9,4,5](12)$} & {$[\mathbf{9}, \mathbf{2}, \mathbf{8}](6)$} & \\
\hline & & {$[\mathbf{9}, \mathbf{4}, \mathbf{6}](3)$} & {$[9,3,6](24)$} & \\
\hline & & {$[\mathbf{6}, \mathbf{2}, \mathbf{5}](18)$} & {$[\mathbf{9}, \mathbf{3}, \mathbf{7}](3)$} & \\
\hline & & & {$[\mathbf{9}, \mathbf{4}, \mathbf{5}](3)$} & \\
\hline \multirow{12}{*}{$\mathbb{F}_{8^{4}}$} & {$[12,8,3](132)$} & {$[12,4,5](12)$} & {$[12, \mathbf{1}, \mathbf{1 2}](6)$} & {$[12, \mathbf{1}, 12](3)$} \\
\hline & {$[\mathbf{1 2}, \mathbf{8}, \mathbf{4}](183)$} & {$[12,4,6](60)$} & {$[12,2,6](3)$} & {$[12,2,6](3)$} \\
\hline & {$[\mathbf{9}, \mathbf{6}, \mathbf{3}](54)$} & {$[\mathbf{1 2}, \mathbf{4}, \mathbf{7}](48)$} & {$[12,2,8](6)$} & {$[12,2,8](6)$} \\
\hline & & {$[12,5,6](21)$} & {$[12,2,10](9)$} & {$[12,2,10](3)$} \\
\hline & & {$[\mathbf{1 2}, \mathbf{5}, \mathbf{7}](12)$} & {$[12,3,6](6)$} & {$[12,3,6](9)$} \\
\hline & & {$[12,6,4](12)$} & {$[12,3,8](3)$} & {$[12,3,8](6)$} \\
\hline & & {$[\mathbf{1 2}, \mathbf{7}, \mathbf{4}](3)$} & {$[\mathbf{1 2}, \mathbf{3}, \mathbf{9}](18)$} & {$[12,3,9](3)$} \\
\hline & & {$[\mathbf{1 2}, \mathbf{8}, \mathbf{4}](72)$} & {$[12,4,7](9)$} & {$[12,4,6](3)$} \\
\hline & & {$[9,3,5](12)$} & {$[12,4,8](3)$} & {$[\mathbf{1 2}, \mathbf{5}, \mathbf{6}](3)$} \\
\hline & & {$[\mathbf{9}, \mathbf{3}, \mathbf{6}](21)$} & {$[12,5,5](3)$} & \\
\hline & & {$[9,4,4](6)$} & {$[\mathbf{1 2}, \mathbf{5}, \mathbf{6}](12)$} & \\
\hline & & {$[\mathbf{9}, \mathbf{5}, \mathbf{4}](3)$} & {$[12,6,5](3)$} & \\
\hline
\end{tabular}

Example 8 Consider $\mathbb{F}_{q}=\mathbb{F}_{4}, \theta(x)=x^{2}$ and $s=20$. This means, we will use elements in $\mathbb{F}_{4^{20}}$ in order to construct codes over $\mathbb{F}_{4}$. We denote by $\gamma$ the generator of $\mathbb{F}_{4^{20}}$ and by $w$ the generator of $\mathbb{F}_{4}$ given by MAGMA. Consider $\alpha=\gamma^{6971}$. The smallest $\mathbb{F}_{2}=\left(\mathbb{F}_{4}\right)^{\theta}$-space $V_{\alpha}$ containing $\{\alpha\}$ which is stable under the generator $\sigma(x)=x^{4}$ has dimension 17 . The skew polynomial corresponding to the Casoratien determinant of a basis of $V_{\alpha}$ is :

$g=X^{17}+w X^{16}+w X^{14}+X^{13}+w^{2} X^{12}+X^{11}+w X^{9}+X^{8}+w X^{7}+w^{2} X^{6}+X^{5}+w X+w$

The bound of $g$ is $f=X^{40}+1$. We obtain a $[40,23,10]$ code over $\mathbb{F}_{4}$. This code improves by one the minimum distance of the previous best known linear code of length 40 and dimension 23 (according to [10]). The upper bound of the minimum distance (here the Griesmer bound) is, in this case, 13.

\section{$5 \quad$ Decoding skew $\mathrm{BCH}$ codes}

These skew codes are built in analogy to BCH codes and it is possible to adapt the classical algorithm to decode these codes. We again adapt the method of ([5], Section 3).

Using the notations of the definition 5 , we consider a $\left(n, q_{0}, t, s, \delta\right)$ skew $\mathrm{BCH}$ code which we denote $\mathcal{C}$. We assume that this code can correct $\Gamma$ errors. 
Consider the codeword $c \in \mathcal{C}$ and the error $e(x)=e_{i_{1}} x^{i_{1}}+\ldots+e_{i_{\gamma}} x^{i_{\gamma}}$. We assume that $\gamma \leq \Gamma$ and denote $c^{\prime}=c+e=\sum_{j=0}^{n} c_{j}^{\prime} x^{j}$ the received word. The question is how to find $e$ knowing $c^{\prime}$.

1. According to ([11], Theorem 1.3.11) we get that the remainder of the right division of $e$ by $X-\alpha^{i}$ for $i=1, \ldots, \delta-1$ is :

$$
A_{i}=\sum_{j=0}^{n-1} e_{j}\left(\alpha^{i}\right)^{[j]}
$$

where $\beta^{[j]}=\beta^{\frac{q_{0}^{i}-1}{q_{0}-1}}$. It is possible to compute $A_{i}$ only with $c^{\prime}$ since the remainder of the right division of $e$ by $X-\alpha^{i}$ is the same as the remainder of the right division of $c^{\prime}$ by $X-\alpha^{i}$ (since $c$ is right divisible by $X-\alpha^{i}$ ). Since $A_{i}$ is also equal to $\sum_{j=0}^{n-1} c_{j}^{\prime}\left(\alpha^{i}\right)^{[j]}$, it can be calculated knowing only $c^{\prime}$. One defines the syndrome polynomial of $e$ as the polynomial :

$$
S(z)=\sum_{k=1}^{\delta-1} A_{i} z^{i-1} \in \mathbb{F}_{q^{s}}[z]
$$

2. One also defines the pseudo-locator polynomial :

$$
\sigma(z)=\prod_{k=1}^{\gamma}\left(1-\alpha^{\left[i_{k}\right]}\right)
$$

and the evaluator polynomial :

$$
w(z)=\sum_{l=1}^{\gamma} e_{i_{l}} \alpha^{\left[i_{l}\right]} \prod_{k \neq l}\left(1-\alpha^{\left[i_{k}\right]} z\right)
$$

3. Knowing $\sigma(z)$ enables us to find the $\left[i_{k}\right]=\frac{q_{0}^{i_{k}}-1}{q_{0}-1} \bmod \left(q^{s}-1\right)$. To do that we have to find $\left[i_{1}\right], \ldots,\left[i_{r}\right]$ so that $\sigma\left(\alpha^{-\left[i_{1}\right]}\right)=, \ldots,=\sigma\left(\alpha^{-\left[i_{r}\right]}\right)=0$. This research can be done by testing $\sigma(x)$ for all $x \in \mathbb{F}_{q^{s}}$. Knowing $\left[i_{k}\right]$ and $w(z)$ enables us to find the coefficients $e_{i_{k}}$ since :

$$
e_{i_{k}}=\alpha^{-\left[i_{k}\right]} w\left(\alpha^{-\left[i_{k}\right]}\right) \prod_{l \neq k}\left(1-\alpha^{\left[i_{l}\right]-\left[i_{k}\right]}\right)
$$

for $k \in\{1, \ldots, \gamma\}$. 
4. We apply Euclid's algorithm to the polynomials $S(z)$ and $z^{\delta-1}$ in $\mathbb{F}_{q^{s}}[z]$. We stop as soon as we find the first remainder of lower degree than $\Gamma$, then we have :

$$
u(z) z^{\delta-1}+v(z) S(z)=r(z)
$$

As in the classical $\mathrm{BCH}$ algorithm, we can prove that $\sigma(z)=v(z) / v(0)$ and $w(z)=$ $r(z) / v(0)$. And if we know $\sigma$ and $w$, we have seen that we can rebuild the error $e$ and find the codeword $c$.

\section{References}

[1] Berrick, A., Keating, M., An introduction to rings and modules, Cambridge Studies in Advanced Mathematics 65, Cambridge University Press (2000).

[2] McDonald, B. R., Finite Rings with Identity, Marcel Dekker Inc. (1974).

[3] Bomboy, R. (1999), Réductibilité des opérateurs aux différences finies: une approche Galois-théorique, Rapport Inria 3735.

[4] Bosma, W., Cannon, J. and Playoust, C. (1997). The Magma Algebra System I: The User Language. Journal of Symbolic Computation, 24, pp. 235-265.

[5] Boucher, D. , Geiselmann, W. and Ulmer, F. (2007), Skew Cyclic Codes, Applicable Algebra in Engineering, Communication and Computing, Vol. 18, pp 379 - 389.

[6] Boucher, D. and Ulmer, F. (2007), Coding with skew polynomial rings, Prépublication IRMAR 08-07, to appear in Journal of Symbolic Computation.

[7] Bronstein, M. and Petkovsek, M. (1994), On Ore Rings, Linear Operators and Factorisation, Programming and Computer Software, 20, pp. 14-26

[8] Gabidulin, E. M. and Kshevetskiy, A. (2005) The new construction of rank codes. 2005 IEEE International Symposium on Information Theory, ISIT'05, pp. 2105 - 2108. -

[9] Gabidulin, E. M. ( 1985) Theory of codes with maximal rank distance. Problems of Information Transmission, vol. 21, pp. 1-12.

[10] Grassl, Markus. Bounds on the minimum distance of linear codes. Online available at http://www.codetables.de. Accessed on 2008-07-02.

[11] Jacobson, N., Finite dimentional algebras over division fields., Springer-Verlag. (1996).

[12] Lidl, R. and Niederreiter, H. Finite Fields., Encyclopedia of Mathematics and its Applications Vol. 20, Amsterdam: Addison-Wesley. (1956).

[13] Lidl, R. and Niederreiter, H. Introduction to finite fields and their applications., Cambridge University Press. (1994). 
[14] Ore, O. (1933). Theory of non-commutative polynomials. Ann. of Math. 34.

[15] Ore, O. (1933). On a Special Class of Polynomials Transactions of the American Mathematical Society, Vol. 35, pp. 559-584

[16] Singer, M. F., Van der Put, M., Galois Theory of difference equations., Springer Lecture Notes in Mathematics, 1666. (1997).

[17] Singer, M. F., Van der Put, M., Galois Theory of linear differencial equations., Grundlehren der mathematischen Wissenschaften, Springer (2003). 